\title{
Evidence for solar shock production of heliospheric near-relativistic and relativistic electron events
}

\author{
Stephen W. Kahler \\ Air Force Research Laboratory/VSBXS, Hanscom AFB, MA 01731, USA \\ email: stephen.kahler@hanscom.af.mil
}

\begin{abstract}
Properties of near-relativistic $(E \gtrsim 30 \mathrm{keV}, \mathrm{NR})$ and relativistic $(E \gtrsim 0.3 \mathrm{MeV})$ electron events produced near the Sun and observed within $1 \mathrm{AU}$ are reviewed. Observations suggest the CME-driven shocks are the sources of many events, but flares are often sources for NR events.
\end{abstract}

Keywords. acceleration of particles, shock waves, Sun: particle emission, flares, CMEs

Transient electron events extend from thermal solar-wind to relativistic energies. Two candidate acceleration processes for these events are MHD shocks driven by fast ( $\gtrsim 1000 \mathrm{~km} / \mathrm{s}$ ) coronal mass ejections (CMEs) and electric field or turbulent wave acceleration during magnetic reconnection in flares. Which process is responsible for observed events?

$N R$ electrons have inferred solar injection onsets delayed by $\sim 10 \mathrm{~min}$ from the flare impulsive phases. The delays, if real, could indicate either a shock or later flare acceleration. Peak electron intensities correlate with CME speeds but also equally well with peak soft and hard X-ray flare fluxes, and most events are not associated with type II burst shocks. Electron spectral variations are ordered by flare soft X-ray flare durations and by CME speeds, but not by the presence or absence of associated CMEs needed to produce shocks. Impulsive ${ }^{3} \mathrm{He}$-rich ion events produced in flares are well associated with electron events, but ion injections may be delayed. Simultaneous ion and electron injections occurred in the big 20 January 2005 event, but both were near the flare impulsive phase and during a very fast CME. Events observed on both Ulysses and $A C E$ during their wide angular separations provide strong evidence for shock acceleration, but most $A C E$ events are not seen at Ulysses, consistent with flare injections. An impulsive component was followed 11 minutes later by a gradual component with a harder spectrum in the 28 October 2003 electron event, suggesting separate flare and shock accelerations and injections. In summary, correlations with CME speeds and type II bursts, associations with gradual SEP events, and large angular distributions suggest that some NR electron events are shock accelerated, but events from impulsive flares are also common.

Relativistic electron injections coincided with flare impulsive phases in Helios observations at $r<0.5 \mathrm{AU}$, but some events consisted of second injections with harder energy spectra. Event onset delays and rise times increase with longitudinal separation from flare sites, and both event rise times to maxima and spectral hardness correlate with CME speeds, as expected for shock acceleration, but event peak intensities correlate with flare $\mathrm{X}$-ray peak fluxes. Two electron events were associated with eruptions with no active region flares. The electrons share the spectral invariance relative to shock locations exhibited by the ions. Similar time-intensity profiles and common e/p ratios in many events suggest that SEPs and electrons are produced together in shocks. Event size distributions match better those of SEPs than of flares, consistent with shocks. 